\title{
Challenges and Opportunities in Classroom Dynamics in an Online as Opposed to an On-Site Class-A Paradigm Shift
}

\section{Bijaya Shrestha*}

Department of Electrical and Computer Engineer, University of Missouri-Rolla, Missouri, 65401, USA

\begin{abstract}
Online class as an emerging mode of education delivery has brought forth new opportunities and challenges on the face of traditional sit-in class. The opportunities are more obvious than the challenges. Compared to our old school format, the lack of a real human being standing in front of the class is the real game changer. The work reported in this article examines these issues.
\end{abstract}

Keywords: Challenges; Opportunities; Paradigm; Online; On-site; State; Operator; Agent of change; Educator; Student; Transformation function

\section{Introduction}

With the advent of computer technology and ease of getting internet access on this side of the digital divide where things can happen and are happening in terms of computer access, a lot of things have changed in the ways education is delivered. We do not necessarily have to have a physical classroom to hold a class anymore. Access to a formal classroom is at anyone's fingertip today as long as there is an access and reliable connectivity to the Internet, and the dollars, of course. Within the system of online education, a classroom does not need to have a physical structure nor a chiming bell to tell if the class has begun or ended. Time and space are no longer the binding factors, at least, not in the sense of our old school ways. Any time, any place, a class can be joined, an exam taken, and a grade earned, within the framework of established schedule, of course. What a concept! Even more interestingly, what a great part of reality! This wave of online education along with everything else that goes on in the online world is spreading all over. Even on the other side of the digital divide, there is a sense of this ever growing urge to climb up the wall and be a part of the digital world in any form or manner. No wonder, the first treasured possession of today's youth in the developing world is the I-phone. Everyone is trying to flow with the "go". Interestingly, online class can be accessed with an I-phone, if need be.

However, all is not as rosy and beautiful as it appears in the first glance. An online class brings a host of challenges, some obvious and some not so much. Among all of these, the lack of a real human being standing in front of the class is the real game changer. Is it a good thing or bad? It depends on who we ask.

An online class is not for everybody and that much is for sure. Some students do better when there is a human being standing in front of them as their professor; unfortunately, in an online class we do not have such a luxury, We could have a video or audio clip of the professor or even have him on Skype or other forums, but it is not the same as a real human being standing in our front. This part of reality seems to matter a lot when we look at online education from the traditional classroom perspective. Along with this, an online class forces us to work with more self-motivation, out of necessity. Such a demand may or may not work well with many online students. Regardless, the dynamics has changed.

\section{Dynamics}

Sometimes and at some venues, where education delivery takes more of a business form, or where the emphasis, knowingly or unknowingly, falls into bringing a large number of students, those too preferably with financial support from places other than the student's own pocket, an unintended dynamics may emerge. Those online classes tend to be taught mechanically, perhaps to free up more time to run several parallel classes. Such a venue will keep any interaction among the students and the teacher to a minimum, which brings an increase in efficiency but at the cost of engagement. In those classes, there would be a discussion thread where the students will have to post a message or two per week and there may be a few automated quizzes that the students will have to take and then at the end of the term, there may be an automated quiz-like final or a proctored final. This painting of a typical online class in those circumstances may appear as being done with rather broad brush but the idea here is to capture the environment than precise details. We may not like to admit but we know there are classes where sometimes the pressure of dollar, henceforth, a desire to see a greater efficiency keeps the class activities sterile, simple, mechanical, and mono-chromatic. The interesting thing here is that some students may like such a situation; the instructor is somewhat aloof which gives them a freedom of detachment. At the same time, some instructors may be very happy to run a course without much personal involvement; the class runs its course, the semester is over, the instructor gets paid; the students receive a grade and during this entire process interaction among each other is kept to a minimum. We all need to think if this is what we envision of a practice that is conducive to true learning? In the lack of clear thinking, deep commitment, and meaningful purpose, sometimes, online teaching could be just a way to make few bucks on the side for some instructors or a quick and clever way to get a degree for some students.

Imparting knowledge to our students in any manner whether by running class online or otherwise involves changing the attitudes of the students at its deepest level. This author is proposing a mathematical formalism to address this issue.

${ }^{*}$ Corresponding author: Bijaya Shrestha, Department of Electrical and Compute Engineer, University of Missouri-Rolla, Missouri, 65401, USA, Tel: (573) 341-6068; E-mail: shrestha@mst.edu

Received June 12, 2015; Accepted July 17, 2015; Published July 28, 2015

Citation: Shrestha B (2015) Challenges and Opportunities in Classroom Dynamics in an Online as Opposed to an On-Site Class-A Paradigm Shift. J Phys Math 6 : 143. doi:10.4172/2090-0902.1000143

Copyright: @ 2015 Shrestha B. This is an open-access article distributed under the terms of the Creative Commons Attribution License, which permits unrestricted use, distribution, and reproduction in any medium, provided the original author and source are credited. 


\section{Mathematical Modeling}

An attitude of a student can be represented by a state function (a mathematical function that represents the state), $\left.\mid \mathrm{s}_{\text {initial }}\right\}$ or $\left.\mid \mathrm{s}_{\mathrm{i}}\right\}$, in short, which may be dependent on host of other parameters, such as educational background, e (in short for educational), cultural background, c, incentives, i, perception, p etc. Given adequate inspiration, motivation, teaching, and mentoring, this state function may be changed to a desired state function, $\left.\mid s_{\text {final }}\right\}$, or $\left.\mid s_{f}\right\}$, in short. Such an agent of change may be represented by an operator, Ō. An agent of change in our context is an educator who effects the change in the state of the attitude of the student. In other words, once the attitude $\left.\mid s_{i}\right\}$ is operated by the operator, $\bar{O}$, it changes the attitude $\left.\mid s_{i}\right\}$ to an attitude $\left.\mid s_{\mathrm{f}}\right\}$. Such a transformation may be expressed mathematically by a transformation function, $\mathrm{T}_{\mathrm{if}}$, as given by, equation (1) below:

$$
T_{i f}=\left\{S_{f}|\bar{O}| S_{i}\right\}, \quad i \neq f \quad \beta_{i f}=1
$$

Our desire here is to realize that equation (1) captures our aspiration as an educator, meaning that the agent of change, O. . will transform $\left.\mid s_{i}\right\}$ to the desired $\left.\mid s_{\mathrm{f}}\right\}$. Here, we also see a new parameter, $\beta_{\text {if }}$. The appendix at the end of this article goes in depth about $\beta_{\text {if }}$. Also, more discussion of the mathematical properties of $T_{\text {if }}$ is given in the appendix which is more of a prelude to a mathematical treatment of this subject matter but at the end of the day, the construct of a meaningful $\mathrm{T}_{\text {if }}$ for an existing set of parameters $\{e, c, i, p, \ldots \ldots\}$ is the ultimate goal to effect the desired change. From the eyes of a traditional sit-in class, the $\mathrm{T}_{\text {if }}$ required in an online class involves a different trajectory. It really needs a shift in the paradigm. The game changer here is that the educator operates in a virtual space. There is somewhat of a misty fog between the teacher and the students. One of the immediate consequences of this is the sense of anonymity (Appendix).

\section{Anonymity-A double edged sword}

A student is invisible or perceived to be invisible in an online class room. This allows students to duck their head from taking responsibility at times or at the other end of the spectrum, allows them to become a bully; either of these attitudes is unhealthy. In particular, the bullying results in the breakdown of integrity and civility in the classroom. In addition to inappropriate, rude, offensive behaviors, other forms of power-play such as, over-participation or domination and underparticipation may surface as well. Possible remedies may be to make expectations clear by spelling out respectful behavior, appropriate conduct, social expectations, and integrity standards in the syllabus itself. Along with this, concerted efforts need to be placed to reduce feelings of detachment. One way to reduce students' feelings of being detached from the course is for the instructor to maintain a consistent social presence in the course, responding and providing information in a timely manner. It should be noted that not all incivility is intentional, though. Sometimes, a lack of understanding of diverse cultures can lead to behaviors that seem intentionally hostile but are not. Also, lack of participation can be the result of a student's apathy or difficulty understanding the course content. Teachers need to go to where students are, and then lead the way to where they need to be. Online or not, a good guidance for instructional design and construct could be had from Bloom's Taxonomy [1] of cognitive operations Although Bloom's taxonomy was developed in the fifties, it is relevant for online learning today Its hierarchy of the six levels of cognitive learning, namely, knowledge, comprehension, application, analysis, synthesis, evaluation applies equally well to the online learning process, from writing courses to coming up with topics for online discussions. With Bloom's Taxonomy, online instructors can define learning objectives and formulate questions and assignments based on each level of cognitive learning just as well. While Bloom's Taxonomy is still the gold standard for instructional design, the peculiarities of online students change the flavor greatly. A successful online education assumes that the online students are involved and active in their courses, are highly motivated, independent, self-starters, organized, disciplined, industrious, creative, and flexible. These are all high expectations. So the question really comes down to the use the taxonomy in a way to accommodate any gap between these high expectations and the reality of the students.

\section{References}

1. Bloom B (1956) Taxonomy of educational objectives. David McKay, New York. 